\title{
miR-483-5p decreases the radiosensitivity of nasopharyngeal carcinoma cells by targeting DAPK1
}

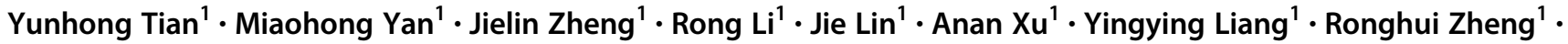 \\ Yawei Yuan ${ }^{1}$
}

Received: 5 June 2018 / Revised: 13 October 2018 / Accepted: 8 November 2018 / Published online: 21 January 2019

(c) United States \& Canadian Academy of Pathology 2019

\begin{abstract}
Recurrence or metastasis resulting from radioresistance are the main challenges for the treatment of nasopharyngeal carcinoma (NPC). A great deal of evidence supports the role of abnormal expression of miRNAs in radioresistance and malignancy. In some cancers, miR-483-5p is associated with inferior disease-specific survival. Therefore, we investigated the role of miR-483-5p in NPC radiosensitivity and the mechanism by which the miR-483-5p affects the radiosensitivity of NPC cells. In this study, we show that the overexpression of miR-483-5p decreases the radiosensitivity of NPC cells in vitro and in vivo. Mechanistically, miR-483-5p exerts these functions by decreasing radiation-induced apoptosis and DNA damage, and by increasing NPC cell colony formation, via targeting death-associated protein kinase 1 (DAPK1). Finally, our results confirm that the upregulation of miR-483-5p is correlated with advanced clinical stage and inferior overall survival of patients with NPC. These findings provide novel insights into our understanding of the molecular mechanisms underlying therapy failure in NPC. Modulation of miR-483-5p and DAPK1 levels may provide a new approach for increasing the radiosensitivity of these tumors.
\end{abstract}

\section{Introduction}

Nasopharyngeal carcinoma (NPC) is a common head and neck malignancy that arises from the superior aspect of the pharyngeal mucosal space, and is found mainly in southern China (population incidence, 25-50 per 100,000) and Southeast Asia [1]. Although progress in the use of radiotherapy has led to advances in the treatment and outcomes of NPC, distant metastasis, recurrence, and radioresistance remain major causes of mortality and morbidity in the advanced stages of the disease, and a challenge for its treatment [2]. Hence, it is important to achieve a deeper understanding of the mechanisms underlying tumor recurrence and resistance to therapies in NPC, since elucidation

These authors contributed equally: Yunhong Tian, Miaohong Yan

Yawei Yuan

yuanyawei2015@outlook.com

1 Department of Radiation Oncology, Affiliated Cancer Hospital \& Institute of Guangzhou Medical University,

Guangzhou, Guangdong Province, People's Republic of China of these could provide strategies to improve treatment efficacy and survival rates.

MicroRNAs (miRNAs), small (20-22 nucleotide) noncoding RNAs molecules, negatively regulate gene expression at the post-transcription level through base-pairing with the $3^{\prime}$-untranslated region (3'-UTR) of mRNA transcripts [3, 4]. Accumulating evidence indicates that miRNAs may act as oncogenes or tumor suppressors by regulating various biological processes, including cell proliferation, metastasis, invasion, and apoptosis. Therefore, miRNAs represent a novel therapeutic target in cancer $[5,6]$. Moreover, many miRNAs have critical roles in tumor radiation responses, which can effectively control tumor radiosensitivity [7-9]. The miRNA, miR-483-5p, is overexpressed in many malignancies, including lung cancer and NPC [10, 11]; however, the role of miR-483-5p in NPC radiosensitivity has not been investigated, and the molecular mechanisms by which miR-483-5p regulates radiosensitivity are unknown. Here, we demonstrate that the overexpression of miR-483-5p can markedly decrease the radiosensitivity of NPC. We also identified deathassociated protein kinase 1 (DAPK1) as a link between miR-483-5p and radioresistance of NPC cells. Hence, we identify a new mechanism of radioresistance in NPC cells, 
which has the potential to lead to new therapeutic strategies.

\section{Materials and methods}

\section{Cell culture}

Three NPC cell lines (CNE-1 and 5-8F) were available at the Cancer Institute of Southern Medical University (Guangzhou, Guangdong Province, China) and were originally purchased from the American Type Culture Collection (ATCC, Manassas, VA, USA). The authenticity of cell lines in our study has been verified with the DNA fingerprinting method in 2013 [12]. This laboratory has never been grown HeLa cells. They were all cultured in RPMI-1640 medium containing 10\% fetal bovine serum (FBS, Gibco, Australia). All cells were cultured in a humidified atmosphere at $37^{\circ} \mathrm{C}$ with $5 \% \mathrm{CO}_{2}$.

\section{Total RNA extraction and quantitative reverse- transcriptase polymerase chain reaction (qRT-PCR)}

Total RNA was extracted from the cells using TRIzol reagent (Invitrogen, Carlsbad, CA, USA) according to the manufacturer's protocol. The PCR primers for miR-483-5p and U6 were purchased from RiBoBio (Guangzhou, Guangdong Province, China). The cDNA library was synthesized using the iScript ${ }^{\mathrm{TM}}$ cDNA synthesis kit (Bio-Rad, Hercules, CA, USA). For mature miRNA quantification, cDNA was generated using specific stem-loop universal primers. qRT-PCR for miRNA and mRNA was performed on an Mx3005P qPCR System (Agilent Technologies, Santa Clara, CA, USA) by using $\mathrm{iQ}^{\mathrm{TM}} \mathrm{SYBR}^{\circledR}$ Green Supermix kit (Bio-Rad). U6 and $\beta$-actin were used as the internal control of miRNA and mRNA, respectively. All reactions were performed in triplicate. The relative amounts of mRNA or miRNA were calculated by using the comparative CT method.

\section{Oligonucleotides and siRNA transfection}

miRNA mimics, scrambled negative control (NC) oligonucleotides, and DAPK1 small interfering RNA (siRNA) were purchased from RiBoBio (Guangzhou). The human miR-483-5p gene was PCR-amplified from genomic DNA and cloned into a lentiviral vector (LV). LV-miR-483-5p was co-transfected with a packaging plasmid into HEK293T cells. Lentiviral particles in the cell culture supernatant were harvested every $24 \mathrm{~h}$ until $72 \mathrm{~h}$ after transfection. Cells were infected with a virus in the presence of $8 \mu \mathrm{g} / \mathrm{ml}$ of polybrene (Sigma-Aldrich, St. Louis, MO, USA). After $48 \mathrm{~h}$ of infection, cells were treated with puromycin for selection. Transfection was performed using Lipofectamine 3000 reagent (Invitrogen) according to the manufacturer's protocol.

\section{Irradiation}

The NPC cells (CNE-1 and 5-8F) after transfection were irradiated at a distance of $100 \mathrm{~cm}$ from the source to the axis using a 6-MV linear accelerator (LINAC; 2300EX; Varian Co., Palo Alto, CA, USA). And the dose rate was $5.0 \mathrm{~Gy} /$ min. X-ray irradiation with a clinically calibrated irradiation field of $10 \mathrm{~cm} \times 10 \mathrm{~cm}$ was performed at the Department of Radiation Oncology, Affiliated Cancer Hospital \& Institute of Guangzhou Medical University (Guangzhou).

\section{Colony formation assay}

According to a previous study, the plating efficiency (PE) of unirradiated controls was calculated using the following formula: number of colonies/number of seeded cells $\times$ 100. The NPC cells (CNE-1 and 5-8F) were transfected with oligonucleotides or siRNA for $48 \mathrm{~h}$. Then cells were seeded on six-well plates in triplicate and exposed to radiation at indicated doses. After incubation at $37{ }^{\circ} \mathrm{C}$ for 9-14 days, the cells were fixed using $100 \%$ methanol and stained with $1 \%$ crystal violet. Colonies containing more than 50 cells were counted under a light microscope. Surviving fractions (SFs) were calculated as the following formula: $\mathrm{SF}=$ number of colonies/number of seeded cells $\times$ PE. The dose-survival curve was fitted based on the single-hit multi-target theory formula: $\mathrm{SF}=$ $1-\left(1-\mathrm{e}^{D / D 0}\right)^{N} ; \log N=D_{\mathrm{q}} / D_{0}$.

\section{Antibodies and western blot analysis}

Proteins from cells or tumor tissues were extracted using radio-immunoprecipitation assay buffer (Sigma-Aldrich) containing a phosphatase inhibitor cocktail and proteinase inhibitor cocktail (Sigma-Aldrich) and quantified using a bicinchoninic acid protein assay kit (Sigma-Aldrich). They were then loaded on sodium dodecyl sulfate (SDS)-polyacrylamide gels at $30 \mu \mathrm{g}$ per lane. After electrophoresis, the proteins were transferred to polyvinylidene fluoride (PVDF, Millipore, MA, USA). Membranes were blocked for $2 \mathrm{~h}$ in $5 \%$ bovine serum albumin (BSA) and incubated overnight at $4{ }^{\circ} \mathrm{C}$ with the SP rabbit polyclonal antibody (1:1000, Santa Cruz Biotechnology, Santa Cruz, CA). After washing the membranes with TBST three times, they were then incubated with horseradish peroxidase (HRP)-conjugated secondary antibody (1:1000, Santa Cruz Biotechnology) for $1 \mathrm{~h}$ at room temperature. Finally, bands were visualized by enhanced chemiluminescence (ECL, Thermo Scientific Pierce, Rockford, IL). 


\section{Flow cytometry analysis of apoptosis}

Irradiated (6 Gy) or control transfected cells were collected $24 \mathrm{~h}$ after irradiation cells undergoing apoptosis were evaluated using the Alexa Fluor 488 Annexin V/Dead Cell Apoptosis Kit (Invitrogen), according to the manufacturer's instructions. Cells were analyzed using a BD FACSCAr$\mathrm{ia}^{\mathrm{TM}}$ flow cytometer (BD Biosciences, CA, USA). Each experiment was performed in triplicate.

\section{Luciferase reporter assays}

The $3^{\prime}$-UTR of DAPKI containing a putative miR-483-5p binding site was amplified from genomic DNA by PCR using the following primers: WT-DAPK1, forward $5^{\prime}$ GGCGGCTCGAGTAGCCCATCCTTCCCTTTG- $3^{\prime}$ and reverse 5'-AATGCGGCCGCTCCTTGGTGACAGCTCC TG- $3^{\prime}$. The amplification product was cloned downstream of the Renilla luciferase reporter gene in the psiCHECK2 dual luciferase reporter plasmid (Promega, Madison, WI, USA) and designated " 3 'UTR-WT". Site-directed mutagenesis of the miR-483-5p target site in the DAPK1 3'-UTR from 5'-CCCGUCU-3' to 5'-GGGCAGA-3' was performed using the Quik-Change ${ }^{\mathrm{TM}}$ Mutagenesis kit (Stratagene, Heidelberg, Germany), with $3^{\prime}$ UTR-WT as the template, and the resulting construct designated " 3 'UTR-MUT". For the luciferase reporter assay, NPC cells or HEK293T cell lines were co-transfected with either NC miRNA or the miR-483-5p mimic, together with a luciferase reporter vector (either $3^{\prime}$ UTR-MUT or $3^{\prime}$ UTR-WT). Cells were lysed using buffer from the Dual-Luciferase Reporter kit (Promega). Luciferase activities were measured $48 \mathrm{~h}$ after transfection, according to the kit manufacturer's instructions, using a Panomics Luminometer (Affymetrix, Santa Clara, CA, USA). Firefly luciferase activity was normalized to that of Renilla luciferase.

\section{Xenograft study}

Female BALB/c nude mice (4-6 weeks old) were purchased from the Model Animal Research Center of Nanjing University (Nanjing, Jiangsu Province, China). All mouse experiments were approved by the animal care and use committee of the Affiliated Cancer Hospital \& Institute of Guangzhou Medical University (Guangzhou). For xenograft tumor assays, a total of $1 \times 10^{7}$ cells stably transfected with vector or LV-miR-483-5p were injected subcutaneously into the right hind limb of mice. Two weeks later, mice whose tumor volumes had reached approximately $200 \mathrm{~mm}^{3}$ were irradiated with a single dose of $10 \mathrm{~Gy}$. Tumor volume was calculated according to the following formula: volume $\left(\mathrm{mm}^{3}\right)=$ length $\times$ width $^{2} / 2$. The expression of DAPK1 was detected by western blot.

\section{Tumor sample immunohistochemistry}

A total of 121 patients with NPC were recruited between January 2008 and December 2010 at the Affiliated Cancer Hospital \& Institute of Guangzhou Medical University (Guangzhou). For the use of these clinical materials for research purposes, prior written informed consent from all the patients and approval from the Ethics Committees of the Affiliated Cancer Hospital \& Institute of Guangzhou Medical University were obtained. Histological classification and clinicopathologic staging of the samples were performed according to the rules of WHO histological classification. All patients were treated with standard curative radiotherapy with or without chemotherapy. DAPK1 was detected using a rabbit polyclonal antibody (Santa Cruz Biotechnology). For clinical samples, all slides were evaluated independently by two investigators who were blinded to the patients' clinical data, using a previously described method. Negative and positive controls were prepared [13].

\section{Statistical analysis}

Statistical analysis was performed using the SPSS statistical package (v15.0) (Abbott Laboratories, North Chicago, IL, USA). In vitro experiments were repeated three times and data are presented as the mean \pm standard deviation (SD). Statistical differences among groups were assessed by oneway analysis of variance (ANOVA). The chi-square test and Fisher's exact test were used to analyze the association of DAPK1 and miR-483-5p expression with other clinicopathological parameters. Kaplan-Meier analysis and the log-rank test were used to evaluate differences in overall survival (OS) rate between groups. $P$-values $\leq 0.05$ were considered statistically significant.

\section{Results}

\section{miR-483-5p decreases the radiosensitivity of NPC cell lines}

To investigate the effect of miR-483-5p on the radiosensitivity of NPC cell lines, we transfected CNE-1 and 5$8 \mathrm{~F}$ cell lines with miR-483-5p mimic or NC, detected the expression of miR-483-5p, and investigated the effect of miR-483-5p on the radiosensitivity of NPC cells. The expression of miR-483-5p significantly increased in CNE-1 and 5-8F cell lines transfected with miR-483-5p. Moreover, after exposure to radiation, the relative survival fractions of CNE-1 and 5-8F cells transfected with miR-483-5p were significantly higher than those of cells transfected with NC. Fitting of dose-survival curves to the single-hit multi-target theory formula also indicated that $\mathrm{CNE}-1$ and $5-8 \mathrm{~F}$ cells 


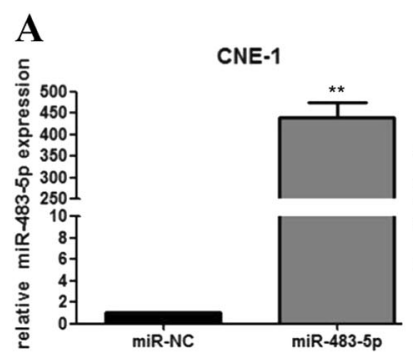

C<smiles>[V]=[V]</smiles>

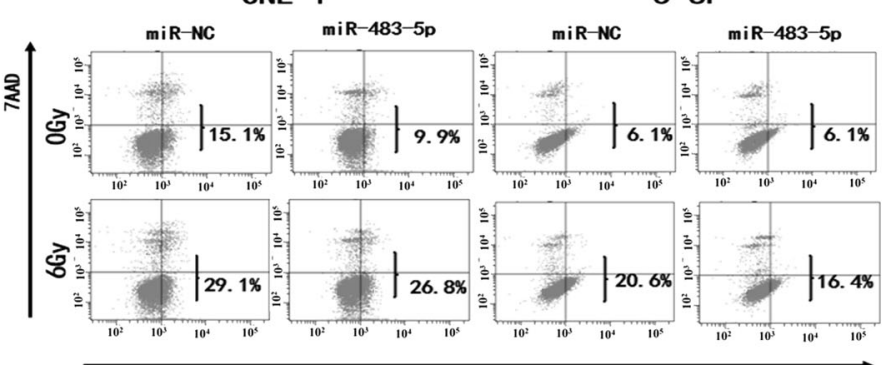

Annexin $V$
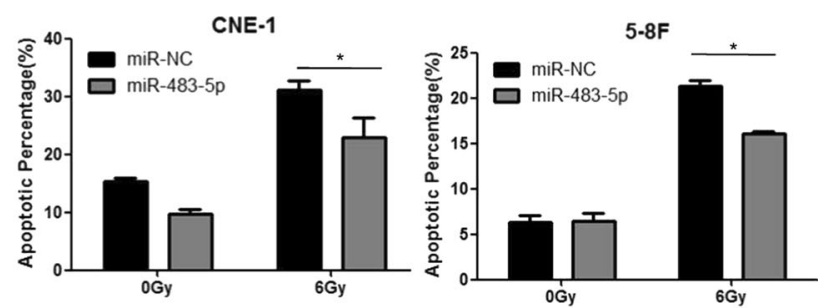

Fig. 1 miR-483-5p decreases the radiosensitivity of NPC cell lines. miR-483-5p mimic (miR-483-5p) or a scrambled negative control (miR-NC) were transfected into CNE-1 (a) or 5-8F (b) cell lines. miR483-5p expression was detected using qPCR $48 \mathrm{~h}$ after transfection. Colony formation assays were performed using NPC cells, and the dose-survival curve was fitted based on the single-hit multi-target

with increased miR-483-5p expression were less sensitive to irradiation (Fig. 1a, b).

Next, we investigated whether miR-483-5p influenced apoptosis after irradiation. Radiation-induced apoptosis was measured by western blot and flow cytometry in both CNE-1 and 5-8F cell lines. The analysis of flow cytometric data showed that the overexpression of miR-483-5p significantly attenuated the percentage of apoptotic cells relative to controls after irradiation (Fig. 1c). Moreover, the expression of cleaved PARP was decreased after irradiation in groups overexpressing miR-483-5p compared with the NC group (Fig. 1d). Finally, we investigated DNA damage by determining $\gamma$-H2AX protein levels which are correlated with DNA double-strand breaks, particularly in cells exposed to irradiation. The results showed that transfection with miR-483-5p mimics led to lower $\gamma-\mathrm{H} 2 \mathrm{AX}$ protein expression after irradiation compared with controls (Fig. 1d). Taken together, these results indicate that the overexpression of miR-483-5p decreases the radiosensitivity of NPC cell lines by decreasing irradiation-

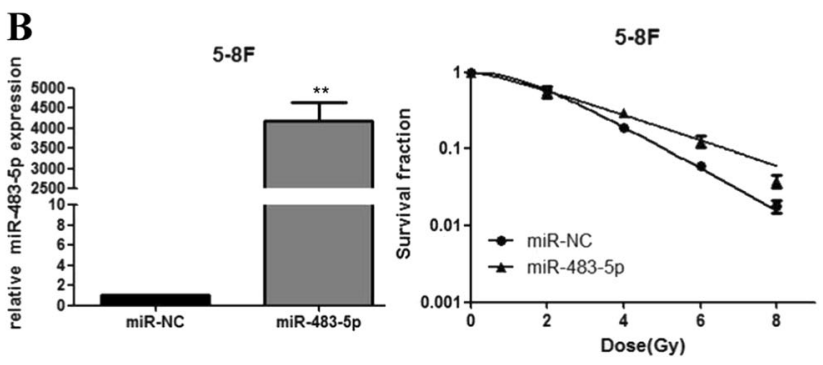

D

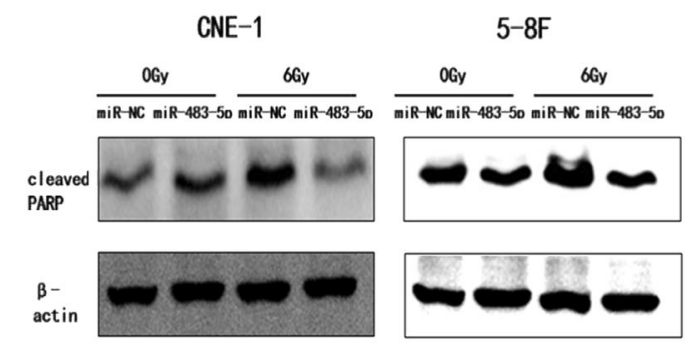

CNE-1

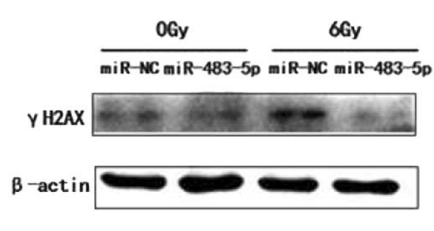

5-8F

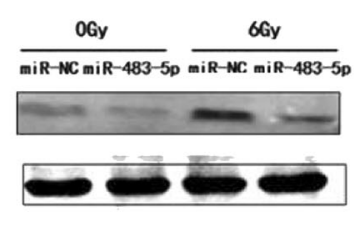

theory formula. c Flow cytometry apoptosis assays were performed using cells treated with or without irradiation. d Expression levels of $\gamma$ $\mathrm{H} 2 \mathrm{AX}$ and cleaved PARP were detected by western blot. Transfected cells were collected $6 \mathrm{~h}$ after irradiation at a dose of $6 \mathrm{~Gy}(n=3 ; * P<$ $0.05)$

induced apoptosis and DNA damage and increasing colony formation ability.

\section{miR-483-5p inhibits the expression of DAPK1 by targeting the DAPK1 $3^{\prime}$-UTR in NPC cells}

To identify the target mRNA of miR-483-5p, miRNA target prediction programs were used including: PicTar (http://www.pictar.org/) and TargetScan (http://www.ta rgetscan.org/). The results showed that miR-483-5p could combine with positions 168-174 of the DAPK1 3'-UTR. To empirically test whether $D A P K 1$ is a target of miR483-5p, we generated luciferase reporter vectors that contained wild-type (3'UTR-WT) or mutant ( $3^{\prime} \mathrm{UTR}$ MUT) miR-483-5p target sequences from the DAPK1 3'UTR (Fig. 2a). We then performed a luciferase reporter assay in CNE-1 and HEK293T cell lines to determine whether DAPK1 was a downstream target of miR-483-5p. As expected, miR-483-5p mimics significantly reduced the luciferase activity generated from the $3^{\prime}$ UTR-WT 
Fig. 2 miR-483-5p inhibits the expression of DAPK1 by targeting the DAPK1 $3^{\prime}$-UTR in NPC cells. a Putative target sites for miR-483-5p binding in the $3^{\prime}$-UTR of DAPK1. Target sequences in the DAPK1 $3^{\prime}$ UTR were mutated. Relative luciferase activity was determined in CNE-1 (b) and HEK293T (c) cells after cotransfection with DAPK1 3' UTR-WT or 3'UTR-MUT plasmids and miR-483-5p mimic or scrambled negative control. d Expression levels of DAPK1 were determined by western blot. e Levels of miR-483-5p and $D A P K 1$ were detected by qPCR in NPC tissue specimens and normalized to those of U6 snRNA and $\beta$-actin, respectively. The correlation between miR-483-5p and $D A P K 1$ expression levels was calculated. Data are presented as means \pm standard error $(n=3$; $* P<0.05, * * P<0.01)$
A

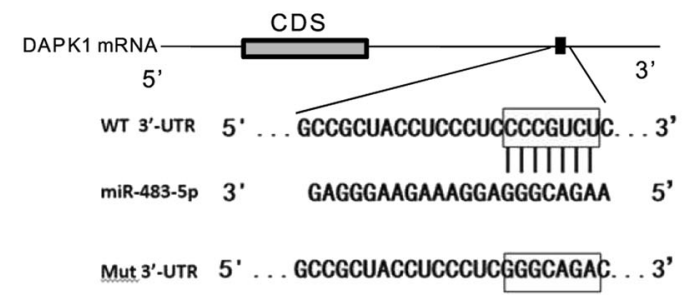

B

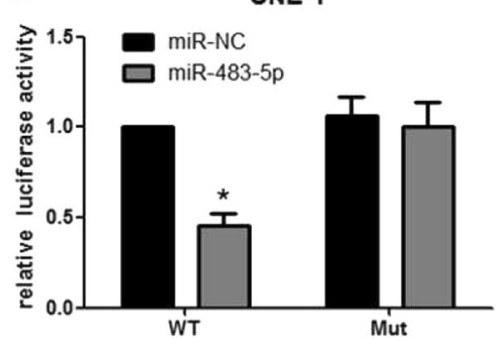

C

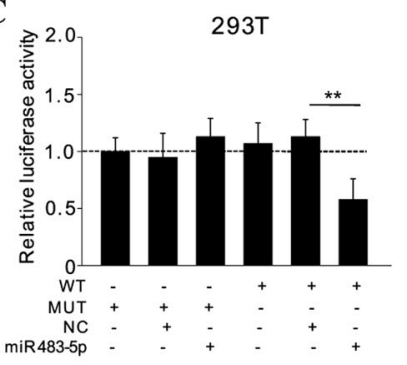

D

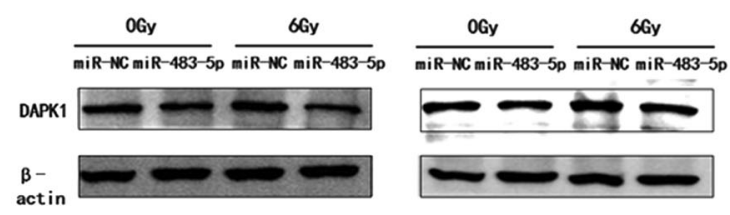

E
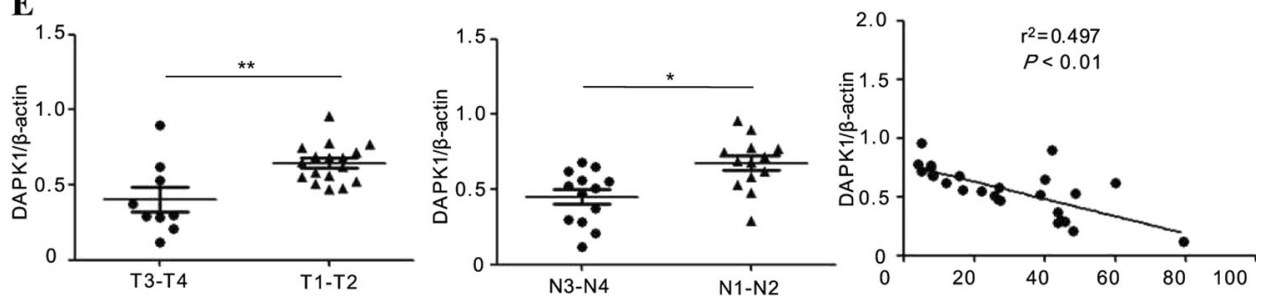

reporter, whereas the relative luciferase activity of the $3^{\prime}$ UTR-MUT reporter was not affected (Fig. 2b, c). Additionally, we found that the overexpression of miR-483-5p could suppress the protein expression of DAPK1 after irradiation (6 Gy) (Fig. 2d). To further confirm the relationship between miR-483-5p and DAPK1, we detected their mRNA expression levels in 26 NPC tissue samples. As shown in Fig. 2e, we observed that down-regulation of $D A P K 1$ expression was correlated with a high degree of tumor spread to regional lymph nodes and advanced tumor stage in NPC. DAPK1 expression was also negatively correlated with miR-483-5p levels. Taken together, these data suggest that miR-483-5p has roles in NPC through direct suppression of $D A P K 1$ expression.

\section{miR-483-5p overexpression and DAPK1 inhibition produce similar changes, which are rescued by ectopic expression of DAPK1 in vitro}

To elucidate whether the effect of miR-483-5p was mediated by DAPK1 in NPC cells, we first examined whether downregulation of DAPK1 elicited similar effects to the expression of miR-483-5p. We transiently transfected CNE1 and 5-8F cells with siDAPK1 or siRNA control and found that inhibition of DAPK1 could significantly attenuate the radiosensitivity of both $\mathrm{CNE}-1$ and $5-8 \mathrm{~F}$ cells (Fig. 3a).
Downregulation of DAPK1 also led to decreased expression of $\gamma-\mathrm{H} 2 \mathrm{AX}$ after irradiation (Fig. 3b). Moreover, apoptosis analysis showed that NPC cells transfected with siDAPK1 underwent less apoptosis after irradiation compared with the control group (Fig. 3c). Overall, these results were similar to the effects of ectopic miR-483-5p expression in NPC cells. Next, we transfected miR-483-5p mimics into NPC cells using a lentivirus vector expressing $D A P K 1$ lacking its $3^{\prime}$-UTR. Flow cytometry analysis indicated that restoring DAPK1 significantly increased the percentage of apoptotic cells suppressed by miR-483-5p after exposure to irradiation (6 Gy) (Fig. 3d). Furthermore, colony formation assays also indicated that the restoration of DAPK1 partially abrogated the inhibitory effects of miR-483-5p on the radiosensitivity of the CNE1 and 5-8F cell lines (Fig. 3e). The results of western blotting analyses also indicated that the expression of $\gamma-\mathrm{H} 2 \mathrm{AX}$ after irradiation was increased by the restoration of DAPK1. Previous reports have indicated that DAPK1 can promote cell apoptosis via extracellular signal-regulated kinase (ERK) pathway [14]. Thus, ERK (1/ 2) protein expression was analyzed, and the results demonstrated that miR-483-5p caused downregulation of DAPK1 and phosphorylated ERK (p-ERK) (1/2) (Fig. 3f). Taken together, these results indicate that miR-483-5p decreases radiosensitivity by directly targeting DAPK1, which interacts with the ERK pathway. 
A
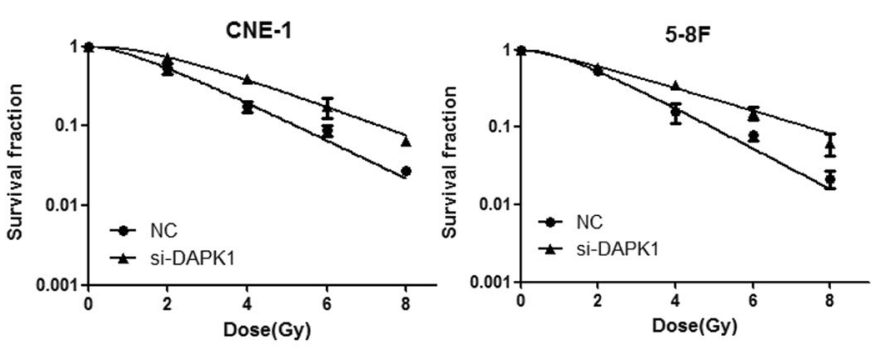

C

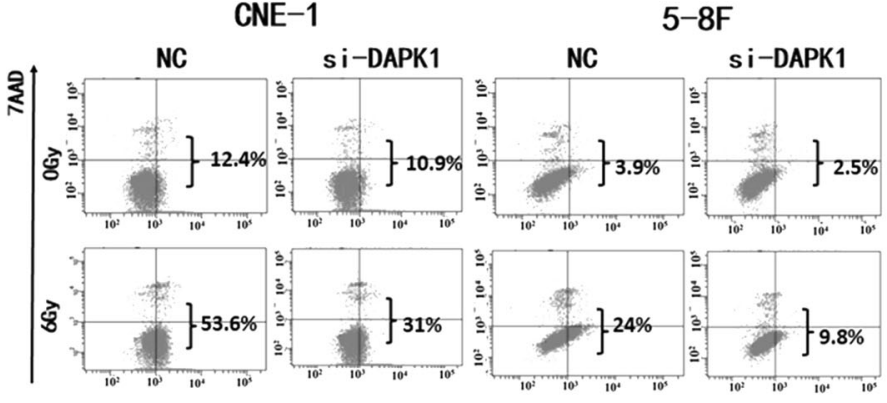

Annexin V

$\mathbf{E}$
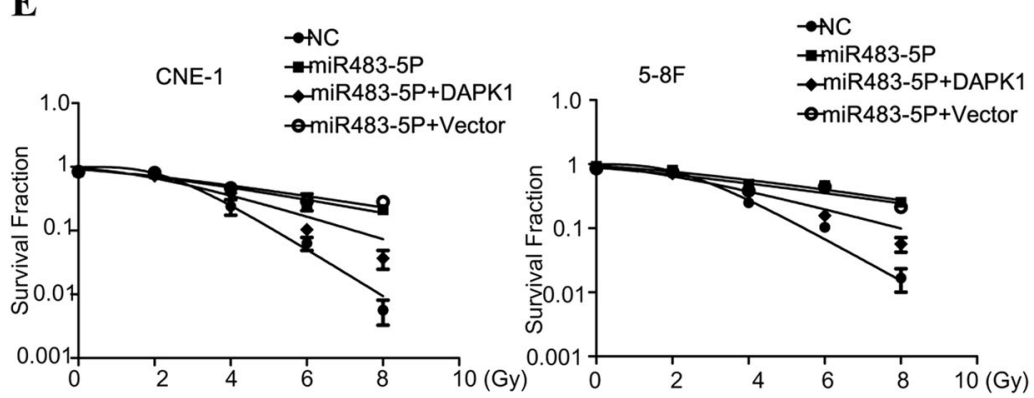

B

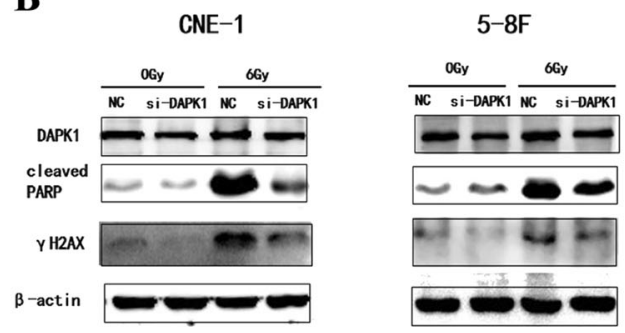

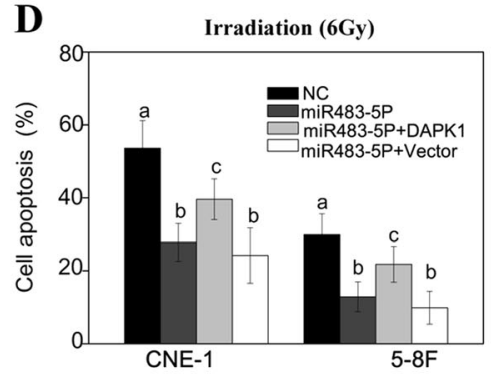

F

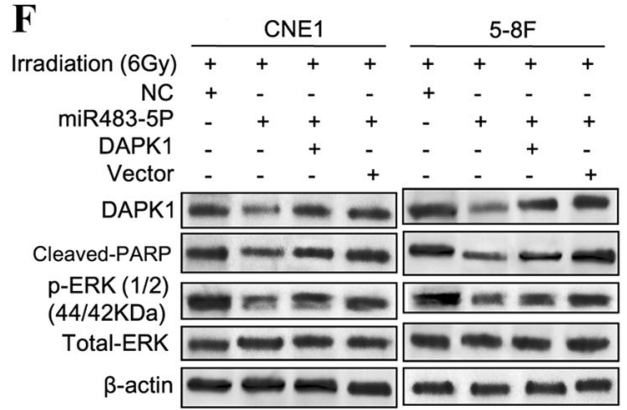

Fig. 3 miR-483-5p overexpression and DAPK1 inhibition resulted in similar changes, which were rescued by ectopic expression of DAPK1 in vitro. a CNE-1 and $5-8 \mathrm{~F}$ cells were transfected with siDAPK1 or negative control (NC) siRNA as indicated. b Expression levels of $\gamma$ H2AX and cleaved PARP were detected by western blotting.

\section{miR-483-5p induces cell resistance to irradiation treatment in vivo}

To determine whether miR-483-5p induces tumor resistance to radiation in vivo, CNE-1 cells overexpressing miR-483-5p or control vector were subcutaneously injected into the right hind limbs of nude mice (Fig. 4a). Fourteen days after the injection, tumor tissues were exposed to a single 10 Gy dose of irradiation. CNE1 cells with stable miR-483-5p expression exhibited enhanced tumor growth in the presence of irradiation (Fig. 4b, c). Measurement of tumor xenograft weights after exposure to irradiation showed that the average tumor weight in the group overexpressing miR-483-5p was significantly greater than that for the control group (Fig. 4d). In addition, our data demonstrate that the in vivo expression of DAPK1 was reduced in xenografts treated with miR-483-5p plus
Apoptosis was measured by flow cytometry assay (c) and quantified (d). e DAPK1 overexpression partially abrogated the inhibitory effect of miR-483-5p on the radiosensitivity of the CNE1 and 5-8F cell lines. f The expression of $\gamma$-H2AX, DAPK1, and p-ERK (1/2) were measured by western blotting after irradiating cells with a dose of $6 \mathrm{~Gy}$

irradiation (Fig. 4e). These results suggested that miR-483$5 \mathrm{p}$ can induce radioresistance in vivo via directly targeting DAPK1.

\section{miR-483-5p overexpression and down-regulated DAPK1 correlates with poor prognosis in NPC}

To determine the clinical significance of miR-483-5p and DAPK1 expression in NPC patients, both were examined in 121 clinical human primary NPC tissues. As shown in Fig. 5a, in DAPK1-positive tissue, positive staining was observed in the tumor cell cytoplasm. Next, the relationships between DAPK1 and miR-483-5p expression and clinicopathological status of patients with NPC were analyzed. As shown in Table 1, miR-483-5p expression was positively associated with advanced $\mathrm{T}, \mathrm{N}$, and clinical NPC stages. DAPK1 expression was also negatively correlated 
A

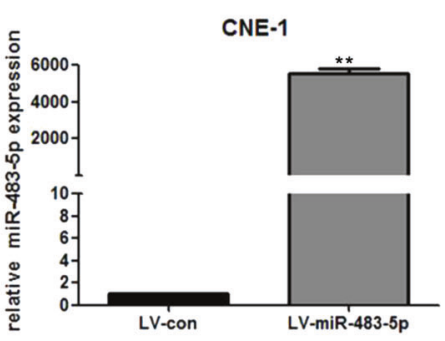

C

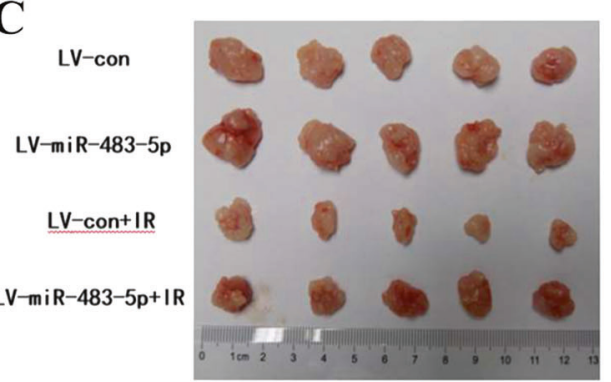

$\mathbf{E}$

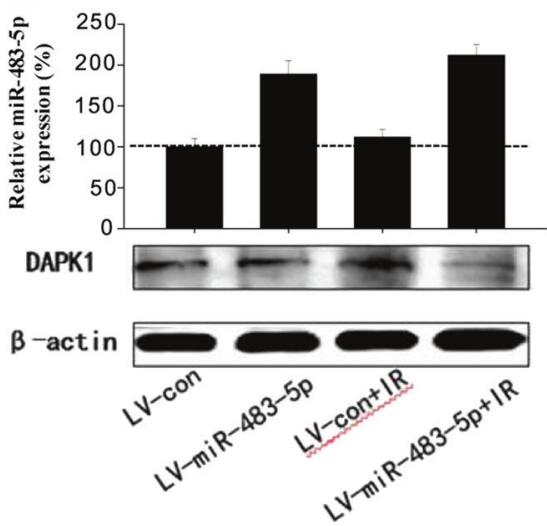

Fig. 4 miR-483-5p induces cell resistance to irradiation treatment in vivo. a The expression level of miR-483-5p in the CNE-1 cell line transfected with LV-miR-483-5p. Nude mice were subcutaneously injected into the right hind legs with $1 \times 10^{7}$ cells overexpressing miR483-5p or a negative control, with or without combined irradiation. b

with $\mathrm{T}$ and N NPC stages; however, no significant correlations were observed between miR-483-5p and DAPK1 expression and sex, age, histologic subtype, or metastasis classification. Moreover, we evaluated the correlation between DAPK1 and miR-483-5p expression and patient OS. Patients with low miR-483-5p or high DAPK1 expression levels had longer OS (Fig. 5b, c), with cumulative 5 -year OS rates of $63.71 \%$ and $70.36 \%$ in patients with low miR-483-5p and high DAPK1 expression, respectively while the equivalent rates in patients with high miR-483-5p and low DAPK1 were only $51.28 \%$ and $41.32 \%$, respectively. In summary, these data suggest that miR-483-5p and its downstream target, DAPK1, are associated with poor prognosis in NPC.

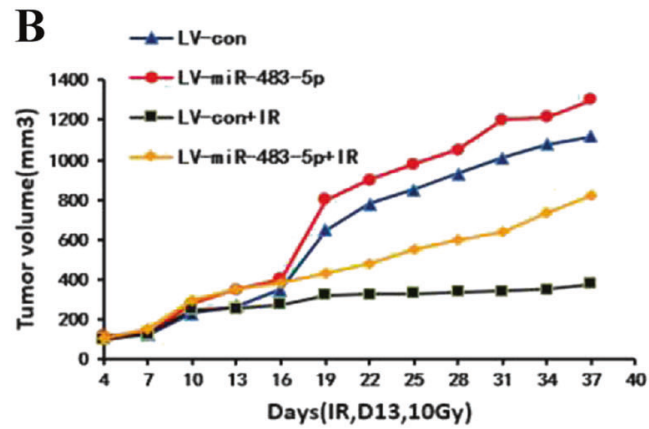

D

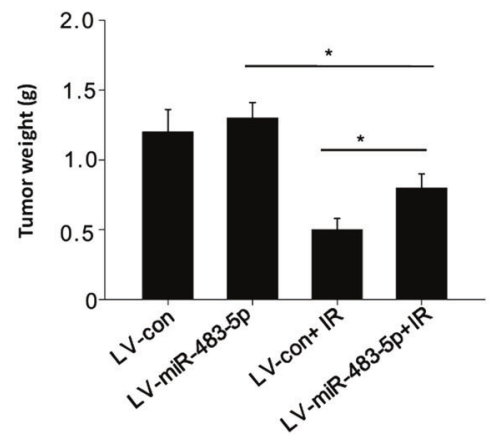

Tumor volume was calculated and presented as tumor growth curves ( $n=5$ per group). $\mathbf{c}$ Representative images of tumors are presented. d Histograms showing the mean tumor weights for each group. e Western blot analysis of DAPK1 extracted from xenografts $(* P<0.05$; $* * P<0.01)$

\section{Discussion}

Radiotherapy has been used as a first-line therapy for NPC; however, patients who develop resistance to radiotherapy during treatment often suffer from recurrence or metastasis [2]. miRNAs, which have crucial roles in tumor progression through modulation of the expression of certain target genes, may act as biomarkers for predicting prognosis and response to radiotherapy in different cancers $[15,16]$. Thus, we investigated the role of miR-483-5p in NPC radiosensitivity and the mechanism by which the miR-483-5p affects radiosensitivity. Our results indicated that miR-483$5 \mathrm{p}$, which is correlated with advanced clinical stage and inferior OS of patients with NPC, can decrease the 
Fig. 5 miR-483-5p overexpression and downregulation of DAPK1 correlates with poor prognosis in NPC. A total of 121 patients with NPC were analyzed by immunohistochemistry. a The expression of DAPK1 was classified as negative when no DAPK1 was detected and positive when there was either complete or incomplete $(>10 \%$ of the tumor cells) staining for DAPK1. Kaplan-Meier survival curves for NPC are plotted for miR-483-5p (b) and DAPK1 (c) expression; differences in survival were evaluated using the log-rank test
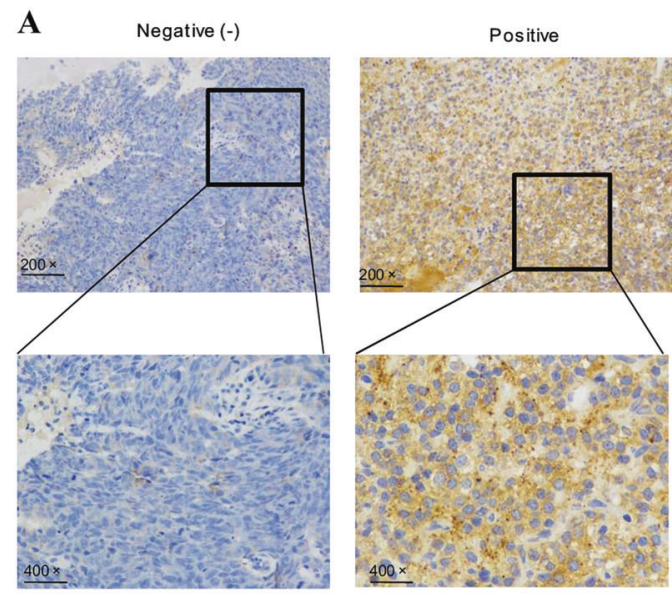
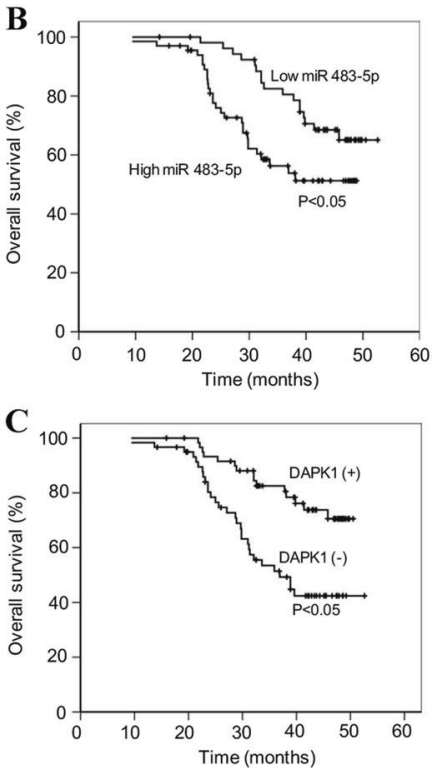

radiosensitivity of NPC cells in vitro and in vivo by targeting the $3^{\prime}$-UTR of DAPK1. Moreover, we found that DAPK1, which is associated with early tumor stage and superior OS in patients with NPC, can markedly decrease the radiosensitivity of NPC cells. These findings provide new insights into the molecular functions of miR-483-5p as well as the role of DAPK1 in radiosensitivity.

miR-483-5p can be dysregulated in, and is associated with, poor disease-specific survival in some cancers [10, 11]. For example, Song et al. reported that miR-483-5p promotes invasion and metastasis of lung adenocarcinoma through targeting RhoGDI1 [10]; Fan et al. found that FIS1 is a direct target of miR-483-5p and that miR-483-5p inhibits mitochondrial fission and the cisplatin sensitivity of tongue squamous cell carcinoma in vitro and in vivo [11]; and Wang et al. found that miR-483-5p was significantly down-regulated in gliomas, whereas the overexpression of miR-483-5p suppressed glioma cell proliferation and induced a G0/G1 arrest by directly targeting ERK1 [17]. Similarly, our results demonstrate that miR-483-5p, which can decrease the radiosensitivity of NPC cells, is correlated with advanced clinical stage and inferior OS in patients with NPC.

Despite the oncogenic and metastasis-promoting role of miR-483-5p implicated in previous reports, the effect of miR-483-5p on radiosensitivity and the molecular mechanisms through which miR-483-5p regulates radiosensitivity are unknown. In this study, we demonstrate, for the first time, that miR-483-5p decreases the radiosensitivity of NPC cells in vitro and in vivo. Moreover, we identified a novel target of miR-483-5p, namely DAPK1. Our results suggested that miR-483-5p directly targets DAPKI by binding to its $3^{\prime}$-UTR region, and significantly decreased
DAPK1 protein expression. To the best of our knowledge, these observations provide the first evidence that miR-483$5 p$ acts as a repressor of DAPK1.

DAPK1 belongs to a family of five serine/threonine kinases that possess tumor suppressive function and also mediate a wide range of cellular processes, including apoptosis [18]. Inactivation of DAPK1 can influence cell death, tumor pathogenesis, and metastasis [19]. Loss of DAPK1 expression occurs in a variety of cancers, mainly via methylation of its promoter. Loss of DAPK1 expression is a crucial role in tumor carcinogenesis and enhances the metastatic potential of cancer cells [20, 21]. DAPK1 is down-regulated or absent in numerous malignant tumors, whereas the over-expression of DAPK1 can induce apoptosis in several cell lines [21, 22]. Consistent with these reports, our data confirm that DAPK1 overexpression is associated with early clinical stage and superior OS in patients with NPC. Although DAPK1 is established as a major influence on apoptosis, its effects on radiosensitivity have not been reported previously. This is the first investigation to demonstrate that DAPK1 is associated with radiosensitivity in cancer cells, particularly NPC. Moreover, we demonstrate that miR-483-5p activates ERK signaling to decrease the radiosensitivity of NPC cells. Dysregulation of the p-ERK pathway has been observed in various forms of cancer and serves as a potential therapeutic target [23].

In conclusion, we found that miR-483-5p, which decreases the radiosensitivity of NPC cells by targeting the 3'-UTR region of $D A P K 1$, was positively associated with advanced tumor stage and positive lymph node metastasis. These findings provide novel insights into the molecular mechanisms underlying the failure to respond to treatment in patients with NPC. Therefore, we speculate that the 
Table 1 Correlations between clinicopathological status and DAPK1 and miR-483-5p expression in NPC

\begin{tabular}{|c|c|c|c|c|c|c|}
\hline \multirow[t]{2}{*}{ Characteristics } & \multicolumn{2}{|l|}{ DAPK1 $(\%)$} & \multirow[t]{2}{*}{$P$ value } & \multicolumn{2}{|c|}{ miR-483-5p (\%) } & \multirow[t]{2}{*}{$P$ value } \\
\hline & $\begin{array}{l}\text { No. of low } \\
\text { expression }\end{array}$ & $\begin{array}{l}\text { No. of high } \\
\text { expression }\end{array}$ & & $\begin{array}{l}\text { No. of low } \\
\text { expression }\end{array}$ & $\begin{array}{l}\text { No. of high } \\
\text { expression }\end{array}$ & \\
\hline \multicolumn{7}{|l|}{ Sex } \\
\hline Male & $31(57.4)$ & $23(42.6)$ & 0.344 & $23(42.6)$ & $31(57.4)$ & 0.202 \\
\hline Female & $45(67.2)$ & $22(32.8)$ & & $37(55.2)$ & $30(44.8)$ & \\
\hline \multicolumn{7}{|l|}{ Age } \\
\hline$<40$ & $26(56.5)$ & $20(43.5)$ & 0.333 & $24(52.2)$ & $22(47.8)$ & 0.71 \\
\hline$\geq 40$ & $50(66.7)$ & $25(33.3)$ & & $36(48.0)$ & $39(52.0)$ & \\
\hline \multicolumn{7}{|c|}{ Histologic subtype } \\
\hline DNKC & $8(80.0)$ & $2(20.0)$ & 0.319 & $6(60.0)$ & $4(40.0)$ & 0.529 \\
\hline UDC & $68(61.3)$ & $43(38.7)$ & & $54(48.6)$ & $57(51.4)$ & \\
\hline T classification & & & & 76 & 45 & \\
\hline $\mathrm{T} 1-2$ & $17(37.0)$ & $29(63.0)$ & $<0.01$ & $34(75.6)$ & $12(24.4)$ & $<0.01$ \\
\hline $\mathrm{T} 3-4$ & $59(78.7)$ & $16(21.3)$ & & $26(34.7)$ & $49(65.3)$ & \\
\hline \multicolumn{7}{|l|}{$\mathrm{N}$ classification } \\
\hline N0-1 & $19(35.2)$ & $35(64.8)$ & $<0.01$ & $35(64.8)$ & $19(35.2)$ & 0.003 \\
\hline N3-4 & $57(85.1)$ & $10(14.9)$ & & $25(37.3)$ & $42(62.7)$ & \\
\hline \multicolumn{7}{|l|}{ M classification } \\
\hline M0 & $64(63.4)$ & 37 (36.6) & 0.804 & $51(50.5)$ & $50(49.5)$ & 0.807 \\
\hline M1 & $12(60.0)$ & $8(40.0)$ & & $9(45.0)$ & $11(55.0)$ & \\
\hline \multicolumn{7}{|l|}{ Clinical stage } \\
\hline I-II & $28(71.8)$ & $11(28.2)$ & 0.227 & $27(69.2)$ & $12(30.8)$ & 0.003 \\
\hline III-IV & $48(58.5)$ & $34(41.5)$ & & $33(39.5)$ & $49(60.5)$ & \\
\hline
\end{tabular}

restoration of miR-483-5p activity represents a potential strategy for application in NPC therapy.

Funding This study was supported by the National Natural Science Foundation of China (Nos. 81502342, 81572964, 81502194, and 81773354); the Funds of Guangzhou Medical College, Guangzhou Key Medical Discipline Construction Project, Guangdong Province, China (Nos. 2016C37 and 2015A23).

\section{Compliance with ethical standards}

Conflict of interest The authors declare that they have no conflict of interest.

Publisher's note: Springer Nature remains neutral with regard to jurisdictional claims in published maps and institutional affiliations.

\section{References}

1. Chen W, Zheng R, Baade PD, Zhang S, Zeng H, Bray F, et al. Cancer statistics in China, 2015. CA Cancer J Clin. 2016;66:115-32.

2. Suarez C, Rodrigo JP, Rinaldo A, Langendijk JA, Shaha AR, Ferlito A. Current treatment options for recurrent nasopharyngeal cancer. Eur Arch Otorhinolaryngol. 2010;267:1811-24.

3. Carthew RW, Sontheimer EJ. Origins and mechanisms of miRNAs and siRNAs. Cell. 2009;136:642-55.
4. Bartel DP. MicroRNAs: target recognition and regulatory functions. Cell. 2009;136:215-33.

5. Leal JA, Lleonart ME. MicroRNAs and cancer stem cells: therapeutic approaches and future perspectives. Cancer Lett. 2013;338:174-83.

6. Bimonte S, Barbieri A, Leongito M, Palma G, Del Vecchio $\mathrm{V}$, Falco M, et al. The role of miRNAs in the regulation of pancreatic cancer stem cells. Stem Cells Int. 2016;2016:8352684.

7. Cortez MA, Valdecanas D, Niknam S, Peltier HJ, Diao L, Giri U, et al. In vivo delivery of miR-34a sensitizes lung tumors to radiation through RAD51 regulation. Mol Ther Nucleic Acids. 2015;4:e270.

8. Moskwa P, Buffa FM, Pan Y, Panchakshari R, Gottipati $\mathrm{P}$, Muschel RJ, et al. miR-182-mediated downregulation of BRCA1 impacts DNA repair and sensitivity to PARP inhibitors. Mol Cell. 2011;41:210-20.

9. Wang J, He J, Su F, Ding N, Hu W, Yao B, et al. Repression of ATR pathway by miR-185 enhances radiation-induced apoptosis and proliferation inhibition. Cell Death Dis. 2013;4:e699.

10. Song Q, Xu Y, Yang C, Chen Z, Jia C, Chen J, et al. miR-483-5p promotes invasion and metastasis of lung adenocarcinoma by targeting RhoGDI1 and ALCAM. Cancer Res. 2014;74:3031-42.

11. Fan S, Chen WX, Lv XB, Tang QL, Sun LJ, Liu BD, et al. miR483-5p determines mitochondrial fission and cisplatin sensitivity in tongue squamous cell carcinoma by targeting FIS1. Cancer Lett. 2015;362:183-91.

12. Wang WJ, Wu SP, Liu JB, Shi YS, Huang X, Zhang QB, et al. $\mathrm{MYC}$ regulation of $\mathrm{CHK} 1$ and $\mathrm{CHK} 2$ promotes radioresistance in a stem cell-like population of nasopharyngeal carcinoma cells. Cancer Res. 2013;73:1219-31.

13. Tian Y, Tian Y, Zhang W, Wei F, Yang J, Luo X, et al. Junctional adhesion molecule-A, an epithelial-mesenchymal transition 
inducer, correlates with metastasis and poor prognosis in human nasopharyngeal cancer. Carcinogenesis. 2015;36:41-8.

14. Chen CH, Wang WJ, Kuo JC, Tsai HC, Lin JR, Chang ZF, et al. Bidirectional signals transduced by DAPK-ERK interaction promote the apoptotic effect of DAPK. EMBO J. 2005;24: 294-304.

15. Zhao L, Lu X, Cao Y. MicroRNA and signal transduction pathways in tumor radiation response. Cell Signal. 2013;25:1625-34.

16. Liu H, Lei C, He Q, Pan Z, Xiao D, Tao Y. Nuclear functions of mammalian microRNAs in gene regulation, immunity and cancer. Mol Cancer. 2018;17:64

17. Wang L, Shi M, Hou S, Ding B, Liu L, Ji X, et al. miR-483-5p suppresses the proliferation of glioma cells via directly targeting ERK1. FEBS Lett. 2012;586:1312-7.

18. Singh P, Ravanan P, Talwar P. Death associated protein kinase 1 (DAPK1): a regulator of apoptosis and autophagy. Front Mol Neurosci. 2016;9:46.
19. Inbal B, Cohen O, Polak-Charcon S, Kopolovic J, Vadai E, Eisenbach L, et al. DAP kinase links the control of apoptosis to metastasis. Nature. 1997;390:180-4.

20. Gade P, Singh AK, Roy SK, Reddy SP, Kalvakolanu DV. Downregulation of the transcriptional mediator subunit Med1 contributes to the loss of expression of metastasis-associated dapk 1 in human cancers and cancer cells. Int J Cancer. 2009;125:1566-74.

21. Cai F, Xiao X, Niu X, Zhong Y. Association between promoter methylation of DAPK gene and HNSCC: a meta-analysis. PLoS ONE. 2017; 12:e0173194.

22. Hoque MO, Feng Q, Toure P, Dem A, Critchlow CW, Hawes $\mathrm{SE}$, et al. Detection of aberrant methylation of four genes in plasma DNA for the detection of breast cancer. J Clin Oncol. 2006;24:4262-9.

23. Samatar AA, Poulikakos PI. Targeting RAS-ERK signalling in cancer: promises and challenges. Nat Rev Drug Discov. 2014;13:928-42. 